\title{
Cost-Effectiveness of CT Screening in the National Lung Screening Trial
}

\author{
William C. Black, M.D., Ilana F. Gareen, Ph.D., Samir S. Soneji, Ph.D., \\ JoRean D. Sicks, M.S., Emmett B. Keeler, Ph.D., Denise R. Aberle, M.D., \\ Arash Naeim, M.D., Timothy R. Church, Ph.D., Gerard A. Silvestri, M.D., \\ Jeremy Gorelick, Ph.D., and Constantine Gatsonis, Ph.D., \\ for the National Lung Screening Trial Research Team*
}

A B STRACT

BACKGROUND

The National Lung Screening Trial (NLST) showed that screening with low-dose computed tomography (CT) as compared with chest radiography reduced lung-cancer mortality. We examined the cost-effectiveness of screening with low-dose CT in the NLST.

\section{METHODS}

We estimated mean life-years, quality-adjusted life-years (QALYs), costs per person, and incremental cost-effectiveness ratios (ICERs) for three alternative strategies: screening with low-dose CT, screening with radiography, and no screening. Estimations of life-years were based on the number of observed deaths that occurred during the trial and the projected survival of persons who were alive at the end of the trial. Quality adjustments were derived from a subgroup of participants who were selected to complete quality-of-life surveys. Costs were based on utilization rates and Medicare reimbursements. We also performed analyses of subgroups defined according to age, sex, smoking history, and risk of lung cancer and performed sensitivity analyses based on several assumptions.

\section{RESULTS}

As compared with no screening, screening with low-dose CT cost an additional $\$ 1,631$ per person ( $95 \%$ confidence interval [CI], 1,557 to 1,709) and provided an additional 0.0316 life-years per person (95\% CI, 0.0154 to 0.0478 ) and 0.0201 QALYs per person ( $95 \%$ CI, 0.0088 to 0.0314 ). The corresponding ICERs were $\$ 52,000$ per life-year gained $(95 \% \mathrm{CI}, 34,000$ to 106,000$)$ and $\$ 81,000$ per QALY gained $(95 \% \mathrm{CI}$, 52,000 to 186,000 ). However, the ICERs varied widely in subgroup and sensitivity analyses.

\section{CONCLUSIONS}

We estimated that screening for lung cancer with low-dose CT would cost $\$ 81,000$ per QALY gained, but we also determined that modest changes in our assumptions would greatly alter this figure. The determination of whether screening outside the trial will be cost-effective will depend on how screening is implemented. (Funded by the National Cancer Institute; NLST ClinicalTrials.gov number, NCT00047385.)

From the Department of Radiology (W.C.B.), Dartmouth Institute for Health Policy and Clinical Practice (S.S.S.), and Norris Cotton Cancer Center (S.S.S.), Geisel School of Medicine at Dartmouth, Dartmouth-Hitchcock Medical Center, Lebanon, NH; Center for Statistical Sciences (I.F.G., J.D.S., J.G., C.G.), Department of Epidemiology (I.F.G.), and Department of Biostatistics (C.G.), Brown University School of Public Health, Providence, RI; Pardee RAND Graduate School, Santa Monica, CA (E.B.K.); Department of Radiological Sciences (D.R.A.) and Divisions of Hematology-Oncology and $\mathrm{Ge}$ riatric Medicine, Department of Medicine (A.N.), David Geffen School of Medicine, University of California at Los Angeles, Los Angeles; Division of Environmental Health Sciences, University of Minnesota School of Public Health, Minneapolis (T.R.C.); and Division of Pulmonary and Critical Care Medicine, Medical University of South Carolina, Charleston (G.A.S.). Address reprint requests to Dr. Black at the Department of Radiology, Geisel School of Medicine at Dartmouth, Dartmouth-Hitchcock Medical Center, Lebanon, NH 03755, or at william.c.black@ hitchcock.org.

*The members of the National Lung Screening Trial Research Team and participating sites are listed in the Supplementary Appendix, available at NEJM.org.

N Engl J Med 2014;371:1793-802. DOI: $10.1056 /$ NEJMoal312547

Copyright @ 2014 Massachusetts Medical Society. 
UNG CANCER IS THE LEADING CAUSE OF cancer-related deaths in the United States ${ }^{1}$; however, until recently, no method of screening had been shown to reduce mortality from lung cancer. The National Lung Screening Trial (NLST) showed that screening with low-dose helical computed tomography (CT) of the chest in patients at high risk for lung cancer was associated with a $20 \%$ reduction in lung-cancer mortality. ${ }^{2}$ Several major medical societies have since recommended screening with low-dose CT for patients with a similarly high risk of lung cancer. ${ }^{3}$ The U.S. Preventive Services Task Force has released a grade $\mathrm{B}$ recommendation for low-dose CT screening, ${ }^{4}$ which means that private insurers must cover the cost of screening. ${ }^{5}$

One major consideration for policymakers is the cost-effectiveness of this screening intervention. ${ }^{6}$ Before publication of the NLST results, several cost-effectiveness analyses reported results ranging from very favorable $e^{7,8}$ to unfavorable., 90 This variation reflected both the uncertainty regarding the effectiveness of screening with low-dose CT for lung cancer and the use of different methods of analysis. At the inception of the NLST, the investigators planned to use the results of the trial to conduct cost-effectiveness analyses and planned the data collection accordingly. ${ }^{2,11-13}$ The primary focus of this report is the cost-effectiveness of screening with low-dose CT as performed in the NLST. The report includes an extensive sensitivity analysis, which is relevant to extrapolation beyond the NLST findings (see Section 1 in the Supplementary Appendix, available with the full text of this article at NEJM.org).

\section{METHODS}

\section{NLST POPULATION}

The NLST was a joint effort of the American College of Radiology Imaging Network (ACRIN) and the Lung Screening Study (LSS). From August 2002 through April 2004, a total of 53,452 persons were enrolled and randomly assigned to undergo three annual screenings with either lowdose CT or chest radiography. ${ }^{2}$ Participants were followed through December 31, 2009. Major eligibility criteria included an age between 55 and 74 years and a smoking history of at least 30 packyears. The study protocol was approved by the institutional review board at each of 33 screening centers (23 ACRIN centers and 10 LSS centers), and written informed consent was obtained from each participant before randomization.

Vital status was based on questionnaires administered semiannually (ACRIN) or annually (LSS); for participants lost to follow-up, status was based on data from the National Death Index. Medical records were obtained from participants who had received positive screening results, a diagnosis of lung cancer, or both, and information related to diagnostic procedures and lungcancer staging and treatment was abstracted with the use of forms that were harmonized across ACRIN and LSS. More detailed information related to diagnostic procedures and lung-cancer treatment was collected from ACRIN sites to inform the cost-effectiveness analysis ${ }^{14,15}$ (see Section 6 in the Supplementary Appendix). In addition, data on health-related quality of life were collected from participants at 16 of the ACRIN screening centers.

\section{SCREENING STRATEGIES AND STUDY DESIGN}

We compared three strategies: screening with lowdose CT, screening with radiography, and no screening. For the two screening strategies, cost and health outcomes were based directly on trial data. For the strategy of no screening, we assumed that health outcomes would be the same as those in the radiography group and that costs would equal those in the radiography group minus the costs of screening examinations and workups for false positive results. We based these assumptions on the results of the Prostate, Lung, Colorectal, and Ovarian (PLCO) Cancer Screening Trial, ${ }^{16}$ which showed no significant reduction in lung-cancer mortality or overdiagnosis in the group randomly assigned to screening with chest radiography.

The analysis conformed to the reference-case recommendations of the U.S. Panel on Cost-Effectiveness in Health and Medicine. ${ }^{12}$ Assuming that screening would affect only the costs and health benefits related to lung-cancer screening and treatment, we conducted the analysis from a societal perspective, in which all health effects and changes in resource use were included. ${ }^{17}$ We considered both a within-trial time horizon (with the effects of screening observed through December 31, 2009) and a lifetime horizon. For each strat- 
egy, we estimated life expectancy and qualityadjusted life expectancy (QALE) from the time of randomization (assumed for the sake of simplicity to be January 1, 2009, for all participants). We reported costs and life-years on a present-value basis, with an annual discount rate of $3 \%$.

\section{LIFE EXPECTANCY}

We assumed that screening did not affect life expectancy in participants who did not receive a diagnosis of lung cancer and that it did not incur costs beyond those involved in the workup for a patient with a positive examination result (with the exception of potentially clinically significant incidental findings and the occurrence of radiation-induced lung cancer in the low-dose CT group after the trial ${ }^{4}$ ) (see Table S7-5 in the Supplementary Appendix). During the trial, there were 116 more lung cancers diagnosed in the CT group than in the radiography group. We also assumed that all these additional cases resulted from overdiagnosis, so that participants in the radiography group without a diagnosis of lung cancer at the end of the trial had the same future risk of lung cancer as those in the CT group. This assumption is justified by the near convergence of lung-cancer incidence in the two groups in the last 3 years of the trial (Table S2-2 and Fig. S2-1 in the Supplementary Appendix). Given these assumptions, the life expectancies of participants without a diagnosis of lung cancer are equal in the two groups (with the exception of the radiation-induced lung cancers occurring only in the low-dose CT group). The life expectancy associated with each screening strategy was equal to a weighted average of the life expectancies of participants with and those without lung cancer.

We excluded 150 of the 53,452 patients who underwent randomization: 100 were lost to follow-up within 1 day after randomization or after their first screening examination (46 in the CT group and 54 in the radiography group), 48 had missing data with respect to lung cancer (i.e., there were not enough data to predict survival [33 in the CT group and 15 in the radiography group]), and 2 were younger than 50 years of age at study entry ( 1 patient in each group). For each of the remaining 53,302 participants (26,642 in the CT group and 26,660 in radiography group), we estimated life-years by adding beyond-trial life-years to within-trial life-years. We calculated within- trial life-years from the date of randomization to the date of death if the patient was deceased (as was the case with 3964 patients); to December 31,2009 , if the patient was alive; or to the latest date on which the patient was known to be alive if data on vital status were missing on December 31, 2009. For the 49,338 participants not known to be deceased on December 31, 2009, we estimated beyond-trial life-years on the basis of their age on the date they were last known to be alive, sex, smoking status at study entry, and lung-cancer stage, if any, using 2009 U.S. Life Tables ${ }^{18}$ adjusted for smoking status and stage-specific annual probabilities of dying from lung cancer (Sections 3 and 4 in the Supplementary Appendix).

We used the observed NLST stage-specific mortality from lung cancer to estimate life expectancy for the first 5 years after diagnosis. For subsequent years, for which NLST data were sparse, we adjusted the observed NLST mortality specific to disease stage to account for the decline in the hazard of death from lung cancer with increased time from the time of diagnosis that was observed in the Surveillance, Epidemiology, and End Results Program ${ }^{19}$ and that has been observed with long-term follow-up of patients with stage I lung cancer detected on CT screening. ${ }^{20}$

To adjust life expectancy for quality, we used utilities derived from the Short Form Health Survey SF-36, ${ }^{21}$ which was administered to 11,696 participants from 16 of 23 ACRIN screening sites. At baseline, the mean utilities (a measure of quality of life on a scale of 0 [death] to 1 [perfect health]) were 0.76 and 0.74 for men and women, respectively, and did not differ by age (Section 5 in the Supplementary Appendix). We also adjusted for lung-cancer stage and time of diagnosis. Because there was no significant difference in utilities between those with false positive screening results and those with true negative screening results, we did not adjust for screening results in the base case. ${ }^{14}$ (The base case reflects several assumptions made to reduce the complexity of our analysis and minimize the use of variables for which there are no reliable estimates. For example, we assumed that all 116 additional cases diagnosed in the CT group were due to overdiagnosis and that there was no reduction in quality of life after a positive screen. See the Supplementary Appendix for further details.) 


\section{costs}

We estimated the expected per-person cost related to lung cancer for the same cohort of 53,302 participants used in the estimation of life expectancy. Total per-person costs were calculated as the sum of direct medical costs and indirect costs. We based direct medical costs on utilization related to the screening examination, diagnostic workup for positive screening results and signs or symptoms of lung cancer, and lungcancer treatment, and we calculated these costs for each participant each year after randomization, using 2009 Medicare prices. ${ }^{22}$ The frequency of screening was based on records indicating adherence to screening with CT or radiography. In the ACRIN subgroup, medical utilization related to diagnostic workup and treatment of lung cancer was obtained directly by means of medical-record abstraction (Section 6 in the Supplementary Appendix). We obtained utilization data from 5133 of the 18,840 participants in the ACRIN subgroup, including almost all those who had a positive screening result, a diagnosis of lung cancer, or both, and imputed costs for LSS participants and ACRIN participants without cost data using variables for which data had been collected for all participants. We based indirect medical costs on time and travel for the participant and caregiver, using 2009 U.S. pricing for hourly earnings ${ }^{23}$ and automobile-mileage reimbursement. ${ }^{24}$ In the base case, we assigned an effective total cost of $\$ 500$ for a participant with at least one potentially clinically significant incidental finding (Section 6.7 in the Supplementary Appendix), and we included medical care (during and after the trial) for lung cancers diagnosed during the trial and for future hypothetical cases of lung cancer induced by radiation from CT screening.

\section{STATISTICAL ANALYSIS}

The three screening strategies were organized according to their baseline costs (from high to low). Incremental costs, life expectancies, QALEs, and incremental cost-effectiveness ratios (ICERs) were calculated for each strategy after the exclusion of any strategy that cost more but provided no benefit as compared with another strategy. We estimated the statistical uncertainty of our results by calculating equal-tail $95 \%$ bootstrap confidence intervals..$^{25,26}$

We performed analyses in subgroups defined according to age (in 5-year age groups), sex, smoking status (current vs. former), and quintiles for the risk of lung cancer that were based on a recently validated model. ${ }^{27}$ To assess the internal validity of our analysis, we dropped three of our base-case assumptions: the assumption that screening with low-dose CT did not affect mortality from causes other than lung cancer, the assumption that all excess cases in the low-dose CT group were overdiagnoses (and instead considering the possibility that up to half of the excess cases were not overdiagnoses), ${ }^{28,29}$ and the assumption that screening with chest radiography was ineffective in reducing lungcancer mortality as compared with no screening (Section 7 in the Supplementary Appendix). In addition, because of the large disparity across groups in the numbers of participants with stage IA non-small-cell lung cancer who were alive at the end of the trial (324 in the CT group vs. 140 in the radiography group) and because of the uncertainty regarding their survival more than 5 years after diagnosis, we repeated the analysis on the basis of optimistic and pessimistic assumptions about their long-term survival (Section 4 in the Supplementary Appendix). We also performed sensitivity analyses of the quality of life after receipt of a positive screening result and after a diagnosis of lung cancer, of radiation-induced lung-cancer deaths, ${ }^{4}$ and of overdiagnosis in the radiography group. Such overdiagnosis would have violated our base-case assumptions with regard to the group that received no screening.

To assess the generalizability of our results, we performed sensitivity analyses of surgical mortality and of the costs of the low-dose CT screening examinations, follow-up CT examinations, surgical resection, chemotherapy, radiation therapy, time and travel, management of incidental findings, and future medical care.

\section{RESULTS}

\section{DIAGNOSES AND DEATHS}

Among the 53,302 participants in our analysis, 1076 in the CT group and 978 in the radiography group received a diagnosis of lung cancer (Table S2-1 in the Supplementary Appendix). Among those in the CT group who had lung cancer, 469 died from lung cancer and 49 died from other causes; among those in the radiography group 


\begin{tabular}{|c|c|c|c|c|}
\hline \multirow[t]{3}{*}{ Time Horizon } & \multicolumn{2}{|c|}{ Life Expectancy } & \multicolumn{2}{|c|}{ Quality-Adjusted Life Expectancy } \\
\hline & $\mathrm{CT}$ & Radiography & $\mathrm{CT}$ & Radiography \\
\hline & \multicolumn{2}{|c|}{ life-yr } & \multicolumn{2}{|c|}{ QALY } \\
\hline Within trial & 5.7846 & 5.7775 & 4.3390 & 4.3351 \\
\hline Participants with lung cancer & 4.9013 & 4.6029 & 3.5603 & 3.3596 \\
\hline Participants without lung cancer & 5.8228 & 5.8228 & 4.3728 & 4.3728 \\
\hline Lifetime & 14.7386 & 14.7071 & 10.9692 & 10.9491 \\
\hline Participants with lung cancer & 8.4792 & 6.8479 & 6.0521 & 4.8981 \\
\hline Participants without lung canceri & 15.0097 & 15.0103 & 11.1821 & 11.1825 \\
\hline
\end{tabular}

* Life-years were discounted at $3 \%$ and are defined as follows: within-trial life-years were calculated from the date of randomization to the date of death if the patient was deceased; to December 31, 2009, if the patient was alive; or to the latest date on which the patient was known to be alive if data on vital status were missing on December 31, 2009. For participants not known to be deceased by that date, beyond-trial life-years were estimated on the basis of the participants' age on the date they were last known to be alive, sex, smoking status at study entry, and lung-cancer stage, if any, with the use of 2009 U.S. Life Tables, ${ }^{18}$ adjusted for smoking status and stage-specific annual probabilities of dying from lung cancer. For further details see Sections 3 and 4 in the Supplementary Appendix. The results for participants who underwent no screening were assumed to be the same as for those who underwent radiographic screening.

$\uparrow$ For participants without lung cancer, outcomes were assumed to be the same for those who underwent CT screening and those receiving radiographic screening except for an adjustment for the occurrence of radiation-induced lung cancer after the trial.

who had lung cancer, 552 died from lung cancer and 35 died from other causes. These numbers represent data from the time of study entry through December 31, 2009.

\section{LIFE EXPECTANCY AND QALE}

Discounted life expectancy and QALE were higher in the CT group than in the radiography group, and the between-group differences were greater when these variables were projected over a lifetime horizon rather than a within-trial horizon because the lifetime horizon accounted for lifeyears saved after the trial (Table 1). For the approximately $4 \%$ of patients with a diagnosis of lung cancer, the incremental life expectancy was 1.6 years.

\section{COSTS AND INCREMENTAL COST-EFFECTIVENESS}

Discounted per-person costs were much higher in the CT group than in the radiography group (Table 2), mainly because of the cost of the screening examination and the much higher Medicare reimbursement for a CT scan of the chest (without the administration of contrast material) than for a chest radiograph (\$285 vs. \$24). ${ }^{22}$ Per-person costs for diagnostic workups and surgery were also higher in the CT group, but the per-person costs of chemotherapy and radiation therapy were lower than those in the radiography group. Future medical costs, which were calculated only in the sensitivity analysis, were slightly higher in the CT group because a

\begin{tabular}{|c|c|c|c|}
\hline \multicolumn{4}{|l|}{ Table 2. Costs per Person.* } \\
\hline Cost & $\begin{array}{c}\text { CT } \\
\text { Screening }\end{array}$ & $\begin{array}{r}\text { Radiographic } \\
\text { Screening } \\
\text { U.S. \$ }\end{array}$ & $\begin{array}{c}\text { No } \\
\text { Screening }\end{array}$ \\
\hline Total & 3,074 & 1,911 & 1,443 \\
\hline Screening & $1,130 \dagger$ & 336 & 0 \\
\hline Workup & 835 & 645 & 512 \\
\hline Treatment & 1,106 & 931 & 931 \\
\hline Surgery & 736 & 470 & 470 \\
\hline Chemotherapy & 282 & 351 & 351 \\
\hline Radiation therapy & 88 & 110 & 110 \\
\hline Radiation-induced lung cancer & 3 & 0 & 0 \\
\hline \multicolumn{4}{|c|}{$\begin{array}{l}\text { * Costs include those for time and travel, which were } \$ 101 \text { for each screening } \\
\text { visit, each workup visit, and each surgical visit. The cost for time and travel } \\
\text { for each radiation therapy visit was } \$ 175 \text { and for each chemotherapy visit, } \\
\$ 381 \text {. } \\
\text { The cost of CT screening includes that of addressing potentially clinically sig- } \\
\text { nificant incidental findings. }\end{array}$} \\
\hline
\end{tabular}




\begin{tabular}{|c|c|c|c|c|c|c|c|c|}
\hline Strategy & Cost & $\begin{array}{c}\text { Life } \\
\text { Expectancy }\end{array}$ & QALE & $\begin{array}{l}\text { Incremental } \\
\text { Costs }{ }^{\prime}\end{array}$ & $\begin{array}{c}\text { Incremental } \\
\text { Life } \\
\text { Expectancy }\end{array}$ & $\begin{array}{l}\text { Incremental } \\
\text { QALE }\end{array}$ & Cost per Life-Yr & Cost per QALY \\
\hline & U.S. \$ & life-yr & $Q A L Y$ & U.S. \$ & life-pr & $Q A L Y$ & \multicolumn{2}{|c|}{ U.S. \$ (95\% CI) } \\
\hline CT screening & 3,074 & 14.7386 & 10.9692 & 1,631 & 0.0316 & 0.0201 & $\begin{array}{c}52,000 \\
(34,000-106,000)\end{array}$ & $\begin{array}{c}81,000 \\
(52,000-186,000)\end{array}$ \\
\hline $\begin{array}{r}\text { Radiographic } \\
\text { screening }\end{array}$ & 1,911 & 14.7071 & 10.9491 & 469 & 0 & 0 & NA & NA \\
\hline No screening & 1,443 & 14.7071 & 10.9491 & - & - & - & - & - \\
\hline
\end{tabular}

* All costs were calculated for the base case, which reflects several assumptions made to reduce the complexity of the analysis and minimize the use of variables for which there are no reliable estimates. NA denotes not applicable, QALE quality-adjusted life expectancy, and QALY quality-adjusted life-year.

$\dagger$ Incremental costs are in reference to the strategy of no screening because the radiography strategy cost more but provided no incremental health benefit as compared with no screening.

$\checkmark$ The cost of the strategy of no screening included the cost of the diagnosis and treatment of lung cancer without low-dose CT or radiographic screening.

higher proportion of participants were still alive at the end of the trial. We assumed that perperson costs in the group undergoing no screening were the same as those in the group undergoing radiography minus the costs for screening

\begin{tabular}{|c|c|c|c|c|}
\hline Characteristic & $\begin{array}{c}\text { No. of } \\
\text { Participants }\end{array}$ & $\begin{array}{l}\text { Incremental } \\
\text { Costs }\end{array}$ & $\begin{array}{c}\text { Incremental } \\
\text { QALYs }\end{array}$ & $\begin{array}{c}\text { Cost per } \\
\text { QALY }\end{array}$ \\
\hline & & U.S. \$ & QALY & U.S. \$ \\
\hline \multicolumn{5}{|l|}{ Sex } \\
\hline Male & 31,446 & 1,683 & 0.0115 & 147,000 \\
\hline Female & 21,856 & 1,557 & 0.0340 & 46,000 \\
\hline \multicolumn{5}{|l|}{ Age at entry } \\
\hline $55-59 \mathrm{yr}$ & 22,773 & 1,541 & 0.0101 & 152,000 \\
\hline $60-64 \mathrm{yr}$ & 16,333 & 1,520 & 0.0320 & 48,000 \\
\hline $65-69 \mathrm{yr}$ & 9,504 & 1,900 & 0.0351 & 54,000 \\
\hline $70-74 \mathrm{yr}$ & 4,685 & 1,905 & 0.0163 & 117,000 \\
\hline \multicolumn{5}{|l|}{ Smoking status } \\
\hline Former & 27,643 & 1,661 & 0.0027 & 615,000 \\
\hline Current & 25,659 & 1,601 & 0.0369 & 43,000 \\
\hline \multicolumn{5}{|l|}{ Risk of lung cancer } \\
\hline First quintile & 10,660 & 1,453 & 0.0086 & 169,000 \\
\hline Second quintile & 10,661 & 1,454 & 0.0118 & 123,000 \\
\hline Third quintile & 10,660 & 1,651 & 0.0061 & 269,000 \\
\hline Fourth quintile & 10,661 & 1,672 & 0.0515 & 32,000 \\
\hline Fifth quintile & 10,660 & 1,851 & 0.0354 & 52,000 \\
\hline
\end{tabular}

and for the workup of false positive screening results.

In our base case, screening with radiography was more expensive than no screening but provided no health benefit (Table 3). As compared with no screening, screening with low-dose CT cost an additional \$1,631 (95\% confidence interval [CI], 1,557 to 1,709) per person and provided an additional 0.0316 life-years per person $(95 \% \mathrm{CI}$, 0.0154 to 0.0478 ) and 0.0201 QALYs per person ( $95 \%$ CI, 0.0088 to 0.0314 ); the corresponding ICERs were $\$ 52,000$ per life-year gained $(95 \% \mathrm{CI}$, 34,000 to 106,000 ) and $\$ 81,000$ per QALY gained (95\% CI, 52,000 to 186,000 ).

\section{SUBGROUP AND SENSITIVITY ANALYSES}

The incremental costs were relatively stable among subgroups, with a range of $\$ 1,453$ to $\$ 1,905$ (Table 4). However, the range for incremental QALYs was much wider $(0.0027$ to 0.0515$)$, with the highest QALY value up to nearly 20 times as high as the lowest; consequently, the range for ICERs was just as wide ( $\$ 32,000$ to $\$ 615,000$ per QALY gained). The ICER was much lower for women than for men ( $\$ 46,000$ vs. $\$ 147,000$ per QALY gained), for current smokers than for former smokers $(\$ 43,000$ vs. $\$ 615,000$ per QALY gained), for the three oldest age groups than for the youngest age group, and for the two quintiles with the highest risk of lung cancer than for the three quintiles with the lowest risk.

Our results were highly sensitive to several of 
our base-case assumptions (Table 5). The ICER fell to $\$ 54,000$ per QALY gained when the reduction in mortality from causes other than lung cancer was included, to $\$ 55,000$ per QALY gained when only half rather than all the excess lung cancers in the CT group were attributed to overdiagnosis, and to $\$ 62,000$ per QALY gained when the risk of death from lung cancer after screening with chest radiography as compared with no screening was reduced to 0.94 . However, the ICER approached or exceeded $\$ 100,000$ per QALY gained when future health care costs were included; when costs for the screening examination, follow-up, or surgery were increased; when the pessimistic expectations of survival with stage IA non-smallcell lung cancer were assumed; and when small reductions in quality of life related to positive screening results and a diagnosis of stage IA lung cancer were included. The ICER rose slightly with the inclusion of deaths from radiation-induced lung cancer.

DISCUSSION

Our base-case estimates of the ICERs for screening with low-dose CT versus no screening $\$ 81,000$ per QALY gained and $\$ 52,000$ per lifeyear gained - fall between the two estimates reported since the intervention was proven effective in the NLST and fall below $\$ 100,000$ per QALY gained, a threshold level that some experts consider to be a reasonable value in the United States. ${ }^{30}$ Much of the difference between our estimate of ICER and the substantially higher estimate reported by McMahon et al. ${ }^{31}$ can be explained by the difference in the number of follow-up CT scans per positive screening examination - approximately one observed in the NLST,,$^{32}$ as compared with four assumed in the study by McMahon et al. Much of the difference between our estimate and the lower estimate reported by Pyenson et $a l .^{33}$ can be explained by their assumption that the reduction in mortality from lung cancer that resulted from screening would be higher than the $20 \%$ observed in the NLST.

The estimated cost-effectiveness of screening with low-dose CT varied widely in the subgroup analysis. Screening with low-dose CT was much more cost-effective among women than among men and among the groups with a higher risk of lung cancer than among those with a lower risk, findings that are consistent with two recent reports showing the greater effectiveness among women $^{34}$ (probably related to different distributions of lung cancer according to histologic type) and among persons at higher risk ${ }^{35}$ in the NLST. Screening with low-dose CT was also more costeffective for current smokers than for former smokers and for the older groups than for the youngest group, findings that are probably due to the higher risk of lung cancer among current smokers and older patients. However, the trends related to age and risk were not uniform, probably because the numbers of lung cancers reported in the subgroups in our study were smaller than the numbers in the total sample.

The ICERs reported in our study were highly sensitive to several of our base-case assumptions. The ICER fell substantially when we excluded our assumptions that CT screening did not affect mortality from causes other than lung cancer, that all excess lung cancers in the CT group were due to overdiagnosis, and that screening with chest radiography was ineffective. The ICER rose substantially when we included future health care costs of survivors that were unrelated to lung cancer, but we did not include these costs in our base case because their inclusion is controversial ${ }^{12}$ and because they have not been included in prior cost-effectiveness analyses of lung-cancer screening. ${ }^{7-10,31,33,35}$ The ICER also rose substantially when we increased the costs of the screening examination, follow-up, and surgery and when we reduced the quality of life related to positive screening results and to a diagnosis of stage IA lung cancer.

We observed no loss of utility in our generic instruments after a report of positive results, a finding that is consistent with another trial, in which false positive screening mammograms were reported $^{36}$; however, distress in response to a positive result (and relief on learning of true negative results) were observed in the NELSON trial (Current Controlled Trials number, ISRCTN63545820) when these emotions were measured with disease-specific instruments measuring quality of life. ${ }^{37}$ All participants in the NLST were provided with detailed information about the frequency and clinical significance of false positive results before study entry, and patients with positive screening results received additional information, which may have reduced their distress. 


\section{Table 5. Results of Sensitivity Analyses.}

\begin{tabular}{|c|c|}
\hline Scenario & $\begin{array}{c}\text { Cost per QALY } \\
\text { U.S. } \$\end{array}$ \\
\hline Base case* & 81,000 \\
\hline Inclusion of non-lung-cancer deaths & 54,000 \\
\hline \multicolumn{2}{|c|}{$\begin{array}{l}\text { Relative risk of screening with radiography vs. no } \\
\text { screening (1.0) }\end{array}$} \\
\hline 0.8 & 40,000 \\
\hline $0.94 \dagger$ & 62,000 \\
\hline 1.1 & 171,000 \\
\hline \multicolumn{2}{|l|}{ No. of future excess cases $(0) ‡$} \\
\hline 29 & 66,000 \\
\hline 58 & 55,000 \\
\hline \multicolumn{2}{|c|}{$\begin{array}{l}\text { Survival for stage IA non-small-cell lung cancer } \\
\text { (intermediate) }\end{array}$} \\
\hline Low & 67,000 \\
\hline High & 108,000 \\
\hline \multicolumn{2}{|l|}{ Cost of screening with low-dose CT (\$285) } \\
\hline 100 & 56,000 \\
\hline 500 & 110,000 \\
\hline \multicolumn{2}{|c|}{$\begin{array}{l}\text { Multiplier for no. of follow-up screenings with } \\
\text { low-dose CT (1) }\end{array}$} \\
\hline 0.5 & 78,000 \\
\hline 5 & 110,000 \\
\hline \multicolumn{2}{|c|}{ Multiplier for cost of surgery $(1=\$ 22,000)$} \\
\hline 0.5 & 73,000 \\
\hline 3 & 114,000 \\
\hline \multicolumn{2}{|l|}{ Surgical mortality (1.2\%) } \\
\hline $0.0 \%$ & 79,000 \\
\hline $8.0 \%$ & 96,000 \\
\hline \multicolumn{2}{|c|}{ Future health care costs ( 0 after CT; 0 after no screening) } \\
\hline$\$ 171,018$ after CT screening & 120,000 \\
\hline \multicolumn{2}{|l|}{$\$ 170,248$ after no screening } \\
\hline \multicolumn{2}{|c|}{ Reduction in quality of life after positive screen (0) } \\
\hline 0.05 & 116,000 \\
\hline \multicolumn{2}{|c|}{$\begin{array}{l}\text { Reduction in quality of life after diagnosis of stage IA lung } \\
\text { cancer }(0.03)\end{array}$} \\
\hline 0.07 & 101,000 \\
\hline \multicolumn{2}{|c|}{$\begin{array}{l}\text { Cost of managing potentially significant incidental } \\
\text { finding }(\$ 500)\end{array}$} \\
\hline 0 & 78,000 \\
\hline$\$ 2,500$ & 96,000 \\
\hline \multicolumn{2}{|c|}{$\begin{array}{l}\text { Radiation-induced lung-cancer deaths per lung-cancer } \\
\text { death prevented }(0.046)\end{array}$} \\
\hline 0 & 79,000 \\
\hline 0.092 & 83,000 \\
\hline
\end{tabular}

* The base case reflects several assumptions made to reduce the complexity of the analysis and minimize the use of variables for which there are no reliable estimates. The base-case value for each variable is given in parentheses.

$\uparrow$ The point estimate is for the subgroup of the Prostate, Lung, Colorectal, and Ovarian Cancer-Screening Trial that was eligible for the National Lung Screening Trial. ${ }^{16}$

$\downarrow$ Future excess cases represent the additional number of lung cancers diagnosed after the trial in the radiography group as compared with the CT group.
Although the NLST results suggest that screening with low-dose CT costs less than $\$ 100,000$ per QALY gained, screening conducted outside the trial may be more costly, depending on the variables that we considered in our sensitivity analyses and on the way in which screening is implemented. One of the most important factors is the cost of the low-dose CT screening examination. Costs could also increase considerably if patient counseling and follow-up are properly accounted for. As the true cost of screening approaches $\$ 500$, the cost becomes prohibitive. The cost could also become prohibitive if the number of follow-up CT scans were to increase. However, the American College of Radiology has developed a reporting system that will raise the positivity threshold that was used in the NLST and could substantially decrease the number of follow-up CTs obtained. ${ }^{38}$

There are several additional limitations to our analysis that deserve consideration. First, we excluded 150 NLST participants from our analysis, 48 of whom had lung cancer but for whom we did not have adequate information to project their survival. Because 47 of these participants were known to be alive at the end of the trial and more of these participants were in the CT group than in the radiography group, their exclusion probably led to a small bias against screening with lowdose CT. Second, we assumed that screening with low-dose CT did not affect smoking status after the time of entry into the NLST. ${ }^{39}$ To the extent that screening caused current smokers to become former smokers, we underestimated the costeffectiveness of screening with low-dose CT. Finally, we did not consider numerous factors that relate to the generalizability of our results outside the NLST, such as the high quality of care provided at NLST screening centers and the stringent selection criteria. However, the Cancer Intervention and Surveillance Modeling Network (CISNET) recently reported an analysis that includes eligibility criteria and screening intervals. ${ }^{40}$

In conclusion, we estimate that screening with low-dose CT for lung cancer as performed in the NLST costs less than $\$ 100,000$ per QALY gained. The determination of whether screening performed outside the trial will be cost-effective will depend on exactly how screening is implemented.

No potential conflict of interest relevant to this article was reported.

Disclosure forms provided by the authors are available with the full text of this article at NEJM.org. 
We thank Dennis G. Fryback, Ph.D., for his advice during the initial planning phase of the cost-effectiveness analysis and the subsequent analysis phase regarding quality of life; Care Communications, which was involved in the planning of the study and the abstraction of the medical records; the following members of the NLST Cost-Effectiveness Analysis Biostatistics Analytic Team for their contributions to the data analysis: Stavroula Chrysanthopoulou, Ph.D., Sarah Demello, M.S., Pratikkumar Desai, M.P.H., and Erin Greco, M.S., all at the ACRIN Biostatistics Center, Brown University, Providence, RI; the NLST-ACRIN Research Team and their research associates, who obtained all the information required for the costeffectiveness analysis; Suzanne B. Lenz, study coordinator for NLST at Dartmouth-Hitchcock Medical Center, for her contributions to the data-collection efforts of the NLST-ACRIN Research Team; the screening-center investigators and the staff of the NLST; and the study participants, whose contributions made this study possible.
REFERENCES

1. Siegel $\mathrm{R}$, Naishadham D, Jemal A. Cancer statistics, 2012. CA Cancer J Clin 2012;62:10-29.

2. Aberle DR, Adams AM, Berg CD, et al. Reduced lung-cancer mortality with lowdose computed tomographic screening. N Engl J Med 2011;365:395-409.

3. Bach PB, Mirkin JN, Oliver TK, et al. Benefits and harms of CT screening for lung cancer: a systematic review. JAMA 2012;307:2418-29.

4. Moyer VA. Screening for lung cancer: U.S. Preventive Services Task Force rec ommendation statement. Ann Intern Med 2014;160:330-8.

5. Patient Protection and Affordable Care Act. August 28, 2013 (http://www .gpo.gov/fdsys/pkg/PLAW-111publ148/ content-detail.html).

6. Sox HC. Better evidence about screening for lung cancer. N Engl J Med 2011; 365:455-7.

7. Marshall D, Simpson KN, Earle CC, Chu C. Potential cost-effectiveness of onetime screening for lung cancer (LC) in high risk cohort. Lung Cancer 2001;32: 227-36.

8. Wisnivesky JP, Mushlin AI, Sicherman $\mathrm{N}$, Henschke C. The cost-effectiveness of low-dose CT screening for lung cancer preliminary results of baseline screening. Chest 2003;124:614-21.

9. Mahadevia PJ, Fleisher LA, Frick KD, Eng J, Goodman SN, Powe NR. Lung cancer screening with helical computed tomography in older adult smokers: a decision and cost-effectiveness analysis. JAMA 2003;289:313-22.

10. Manser R, Dalton A, Carter R, Byrnes G, Elwood M, Campbell DA. Cost-effectiveness analysis of screening for lung cancer with low dose spiral CT (computed tomography) in the Australian setting. Lung Cancer 2005;48:171-85.

11. Aberle DR, Berg CD, Black WC, et al. The National Lung Screening Trial: overview and study design. Radiology 2011; 258:243-53.

12. Gold MR, Siegel JE, Russell LB, Weinstein MC, eds. Cost-effectiveness in health and medicine. Oxford, England: Oxford University Press, 1996.

13. Ramsey S, Willke R, Briggs A, et al. Good research practices for cost-effectiveness analysis alongside clinical trials: the ISPOR RCT-CEA Task Force report. Value Health 2005;8:521-33.

14. Gareen IF, Duan F, Greco EM, et al. Impact of lung cancer screening results on participant health-related quality of life and state anxiety in the National Lung Screening Trial. Cancer 2014; 10.1002/ cncr.28833.

15. Gareen IF, Sicks JD, Molene D, et al. Identifying and collecting pertinent medical records for centralized abstraction in a multi-center randomized clinical trial: the model used by the American College of Radiology arm of the National Lung Screening Trial. Contemporary Clinical Trials 2013;24:36-44.

16. Oken MM, Hocking WG, Kvale PA, et al. Screening by chest radiograph and lung cancer mortality: the Prostate, Lung, Colorectal, and Ovarian (PLCO) randomized trial. JAMA 2011;306:1865-73.

17. Russell LB, Gold MR, Siegel JE, Daniels N, Weinstein MC. The role of cost-effectiveness analysis in health and medicine. JAMA 1996;276:1172-7.

18. Arias E. United States life tables, 2009. Natl Vital Statcs Rep 2014;62(7):1-63.

19. Surveillance, Epidemiology, and End Results (SEER) Program. SEER ${ }^{\star}$ Stat Database: Incidence - SEER 18 Regs Research Data + Hurricane Katrina Impacted Louisiana Cases, Nov 2012 Sub (1973-2010 varying), April 2013 (http://www.seer .cancer.gov/data/seerstat/nov2012).

20. Henschke CI, Yankelevitz DF, Libby DM, Pasmantier MW, Smith JP, Miettinen OS. Survival of patients with stage I lung cancer detected on CT screening. N Engl J Med 2006;355:1763-71.

21. Brazier J, Roberts J, Deverill M. The estimation of a preference-based measure of health from the SF-36. J Health Econ 2002;21:271-92.

22. Centers for Medicare and Medicaid Services. Physician Fee Schedule look-up tool. March 1, 2011 (http://www.cms.hhs .gov/pfslookup/02_PFSsearch.asp).

23. Department of Labor. Employer costs for employee compensation - December 2009. March 10, 2010 (http://www.bls .gov/news.release/archives/ecec_03102010 .pdf).

24. General Services Administration. Privately owned vehicle (POV) mileage reimbursement rates. 2012.
25. Briggs $\mathrm{AH}$, Wonderling DE, Mooney CZ. Pulling cost-effectiveness analysis up by its bootstraps: a non-parametric approach to confidence interval estimation. Health Econ 1997;6:327-40.

26. Stinnett AA. Adjusting for bias in $\mathrm{C} / \mathrm{E}$ ratio estimates. Health Econ 1996;5:4702.

27. Tammemägi MC, Katki HA, Hocking WG, et al. Selection criteria for lung-cancer screening. N Engl J Med 2013;368:72836.

28. Welch HG, Black WC. Overdiagnosis in cancer. J Natl Cancer Inst 2010;102:60513.

29. Veronesi G, Maisonneuve P, Bellomi $\mathrm{M}$, et al. Estimating overdiagnosis in lowdose computed tomography screening for lung cancer: a cohort study. Ann Intern Med 2012;157:776-84.

30. Neumann PJ, Cohen JT, Weinstein MC. Updating cost-effectiveness - the curious resilience of the $\$ 50,000$-per-QALY threshold. N Engl J Med 2014;371:796-7.

31. McMahon PM, Kong CY, Bouzan C, et al. Cost-effectiveness of computed tomography screening for lung cancer in the United States. J Thorac Oncol 2011;6: 1841-8.

32. Church TR, Black WC, Aberle DR, et al. Results of initial low-dose computed tomographic screening for lung cancer. $\mathrm{N}$ Engl J Med 2013;368:1980-91.

33. Pyenson BS, Sander MS, Jiang Y, Kahn $\mathrm{H}$, Mulshine JL. An actuarial analysis shows that offering lung cancer screening as an insurance benefit would save lives at relatively low cost. Health Aff (Millwood) 2012;31:770-9.

34. Pinsky PF, Church TR, Izmirlian G, Kramer BS. The National Lung Screening Trial: results stratified by demographics, smoking history, and lung cancer histology. Cancer 2013;119:3976-83.

35. Kovalchik SA, Tammemagi M, Berg $\mathrm{CD}$, et al. Targeting of low-dose CT screening according to the risk of lung-cancer death. N Engl J Med 2013;369:245-54.

36. Tosteson AN, Fryback DG, Hammond $\mathrm{CS}$, et al. Consequences of false-positive screening mammograms. JAMA Intern Med 2014;174:954-61.

37. van den Bergh KA, Essink-Bot ML, Borsboom GJ, et al. Short-term healthrelated quality of life consequences in a 
lung cancer CT screening trial (NELSON). Br J Cancer 2010;102:27-34.

38. American College of Radiology. Lung Imaging Reporting and Data System (LungRADS). April 29, 2014 (http://www.acr.org/ Quality-Safety/Resources/LungRADS).

39. van der Aalst CM, van Klaveren RJ, van den Bergh KA, Willemsen MC, de Koning HJ. The impact of a lung cancer computed tomography screening result on smoking abstinence. Eur Respir J 2011; 37:1466-73.

40. de Koning HJ, Meza R, Plevritis SK, et al. Benefits and harms of computed to- mography lung cancer screening strategies: a comparative modeling study for the U.S. Preventive Services Task Force. Ann Intern Med 2014;160:311-20.

Copyright @) 2014 Massachusetts Medical Society. 